\title{
ARTICLE
}

\section{Administration frequency as well as dosage of PTH are associated with development of cortical porosity in ovariectomized rats}

\author{
Aya Takakura ${ }^{1,2}$, Ji-Won Lee ${ }^{3}$, Kyoko Hirano ${ }^{1}$, Yukihiro Isogai ${ }^{1}$, Toshinori Ishizuya ${ }^{1}$,
} Ryoko Takao-Kawabata ${ }^{1}$ and Tadahiro Iimura ${ }^{2,3,4,5}$

To investigate whether the administration frequency of parathyroid hormone (PTH) is associated with the development of cortical porosity, this study established $\mathbf{1 5}$ dosage regimens of teriparatide [human PTH (1-34), TPTD] with four distinct concentrations and four distinct administration frequencies of TPTD to 16-week-old ovariectomized rats. Our analyses demonstrated that the bone mineral density, mechanical properties, and bone turnover were associated with the total amount of TPTD administered. Our observations further revealed that the cortical porosity was markedly developed as a result of an increased administration frequency with a lower concentration of total TPTD administration in our setting, although the highest concentration also induced cortical porosity. Deconvolution fluorescence tiling imaging on calcein-labeled undecalcified bone sections also demonstrated the development of cortical porosity to be closely associated with the bone site where periosteal bone formation took place. This site-specific cortical porosity involved intracortical bone resorption and an increased number and proximity of osteocytic lacunae, occasionally causing fused lacunae. Taken together, these findings suggested the involvement of local distinctions in the rate of bone growth that may be related to the site-specific mechanical properties in the development of cortical porosity induced by frequent and/or high doses of TPTD.

Bone Research (2017) 5, 17002; doi:10.1038/boneres.2017.2; published online: 25 April 2017

\section{INTRODUCTION}

The effects of parathyroid hormone (PTH) on rat bone metabolism were first observed in the early 1900s. ${ }^{1-3}$ Since the achievement of the chemical synthesis of human PTH (1-34) in the 1970s, studies have attempted to define the detailed mechanism of this hormonal action. ${ }^{4}$ The anabolic effect of PTH on the skeleton in post-menopausal osteoporosis reported by Reeve et al ${ }^{5}$ was the first clinical application.

PTH is well known to exert its anabolic effects on bone through promoting the proliferation, differentiation and survival of bone-forming osteoblasts, which concomitantly induce the differentiation of osteoclasts, thereby promoting bone resorption. ${ }^{6}$ The balance between these anabolic and catabolic actions of PTH administration is thought to be defined by the blood concentration of PTH, although the exact molecular and cellular mechanisms underlying these phenomena remain controversial. Bone formation and resorption are preferentially promoted when blood PTH level are intermittently and persistently high, respectively. In other words, the effect of PTH on bone is dosage regimen-dependent. The intermittent exposure of PTH increases bone formation over resorption, whereas the continuous infusion of PTH stimulates bone resorption over formation, causing a net loss in bone mass. $^{7-8}$

\footnotetext{
'Pharmaceuticals Research Center, Asahi Kasei Pharma Corporation, Shizuoka, Japan; ${ }^{2}$ Graduate School of Medicine, Division of Analytical BioMedicine, Ehime University, Ehime, Japan; ${ }^{3}$ Division of Bio-Imaging, Proteo-Science Center (PROS), Ehime University, Ehime, Japan; ${ }^{4}$ Division of Analytical Bio-Medicine, Advanced Research Support Center (ADRES), Ehime University, Ehime, Japan and ${ }^{5}$ Artificial Joint Integrated Center, Ehime University Hospital, Ehime, Japan Correspondence: Tadahiro limura (iimura@m.ehime-u.ac.jp) or Ryoko Takao-Kawabata (takao.rb@om.asahi-kasei.co.jp) Received: 10 August 2016; Revised: 1 November 2016; Accepted: 28 November 2016
} 
PTH binds to its cell surface receptor PTH1R, which is expressed in osteoblasts and their precursors, chondrocytes, and vascular smooth muscle cells. Recent findings have demonstrated that PTHIR-mediated signaling causes two distinct processes: a canonical G protein-coupled receptor (GPCR)-cyclic AMP (CAMP) pathway and an endocytosis-mediated pathway. 'The former induces a transient CAMP response derived from signaling complexes localized at the plasma membrane, whereas the latter induces a prolonged CAMP response mediated by molecular complexes associated within the endosome. Studies using PTH analogs in vivo have suggested that selective stimulation of the endosome-mediated long signal and the prolonged half-life of PTH in the blood are favorable for inducing hypercalcemia. $^{10-11}$ However, the association between the temporal variations in PTH-mediated CAMP signaling and the downstream cellular responses that promote bone anabolism and catabolism remains unclear.

Cortical porosity occurs mainly by the stimulation of intracortical remodeling, thus causing thinner cortices, as clinically observed in elderly women and women with hyperparathyroidism. ${ }^{12-14}$ Recent findings have emphasized the relevance of cortical porosity as a determinant of bone fragility that is highly associated with fracture risk, although clinical and basic studies have tended to focus on vertebral fractures and trabecular bone loss. ${ }^{15-18}$ The continuous stimulation of PTH has been reported to induce the early development of cortical porosity. ${ }^{19}$ Once-daily administration of teriparatide [human PTH(1-34), TPTD] has been found to increase cortical porosity in clinical studies ${ }^{20}$ and animal studies using rabbits ${ }^{21}$ and dogs. ${ }^{22}$ These findings suggest that the dosage regimen of PTH affects not only the net bone mass but also the microarchitecture of bone, including cortical porosity, which may in turn affect the pharmacological action of PTH with respect to preventing the risk of bone fracture. Members of our research group have recently reported that a long-term and lower frequency (three times weekly) TPTD administration in ovariectomized (OVX) rats improves the bone density, microarchitecture, and mechanical properties of bone without obvious stimulation of bone resorption. ${ }^{23}$ However, there is little information in the literature concerning the development of cortical porosity at any particular frequency or dosage of PTH administration, although such information would provide relevant information to clinical settings involving $\mathrm{PTH}$ treatment.

In this study, we established 15 regimens with four distinct concentrations and four distinct administration frequencies of TPTD administration to 13-week-old OVX rats as a standard post-menopausal osteoporosis animal model for PTH examination ${ }^{23-25}$ and then analyzed the bone properties, focusing on the development of cortical porosity.

\section{MATERIALS AND METHODS}

Animals

Eleven-week-old, sexually mature female Sprague-Dawley rats (Charles River, Kanagawa, Japan) were used. The rats were maintained under a 12-h light/dark cycle and given unrestricted access to tap water and a standard diet containing $1.2 \%$ calcium, $0.9 \%$ phosphorus, $22.0 \%$ protein, and 6.2 IU vitamin D3 per gram (CRF-1; Oriental Yeast, Tokyo, Japan). The animals were allowed to acclimate to their environment for 2 weeks before the start of the experiment. The experimental protocols were approved by the experimental animal ethics committee at Asahi Kasei Pharma Corporation and were conducted in accordance with the guidelines concerning the management and handling of experimental animals.

\section{Experimental design}

At 13 weeks of age, sexually mature rats were divided into 15 groups (Table 1) and subjected to either bilateral ovariectomy or sham surgery under anesthesia comprising intraperitoneal injection with $\sim 48 \mathrm{mg} \cdot \mathrm{kg}^{-1}$ ketamine hydrochloride and $4 \mathrm{mg} \cdot \mathrm{kg}^{-1}$ xylazine, as previously described. ${ }^{23,26}$ For the sham surgery, $~ 10-\mathrm{mm}$-long incisions were made on the back of each rat, as in the ovariectomy, and the ovaries were exposed and then replaced.

Three weeks after the surgery, at 16 weeks of age, the rats were subcutaneously injected with 6 or $30 \mu \mathrm{g} \cdot \mathrm{kg}^{-1}$ of chemically synthetized TPTD acetate (Asahi Kasei Pharma Corporation, Tokyo, Japan) three times a week (W3 groups); $1.2,6$ or $30 \mu \mathrm{g} \cdot \mathrm{kg}^{-1}$ of TPTD once a day

Table 1. The regimen settings of teriparatide [human $\mathrm{PTH}(1-34)$, TPTD] administration in ovariectomized rats

\begin{tabular}{|c|c|c|c|c|c|c|c|}
\hline \multicolumn{2}{|c|}{ Group } & \multirow{3}{*}{$\begin{array}{l}\text { Operation } \\
\text { Sham }\end{array}$} & \multirow{3}{*}{$\begin{array}{l}\text { Treatment } \\
\text { Vehicle }\end{array}$} & \multicolumn{2}{|c|}{ Dose } & \multirow[t]{2}{*}{ Frequency } & \multirow[t]{2}{*}{$n$} \\
\hline & & & & $\left(\mu \mathrm{g} \cdot \mathrm{kg}^{-1}\right)$ & $\begin{array}{c}\left(\mu \mathrm{g} \cdot \mathrm{kg}^{-1}\right. \\
\text { per week) }\end{array}$ & & \\
\hline 1 & Sham & & & - & - & 3/week & 5 \\
\hline 2 & W3V & ovx & Vehicle & - & - & 3/week & 5 \\
\hline 3 & W3M & ovx & TPTD & 6 & 18 & 3/week & 5 \\
\hline 4 & W3H & ovx & TPTD & 30 & 90 & 3/week & 5 \\
\hline 5 & DIV & ovx & Vehicle & - & - & 1/day & 5 \\
\hline 6 & DIL & ovx & TPTD & 1.2 & 8.4 & 1/day & 5 \\
\hline 7 & DIM & ovx & TPTD & 6 & 42 & 1/day & 5 \\
\hline 8 & $\mathrm{DlH}$ & OVX & TPTD & 30 & 210 & 1/day & 5 \\
\hline 9 & $\mathrm{D} 2 \mathrm{~V}$ & ovx & Vehicle & - & - & 2/day & 5 \\
\hline 10 & D2L & OVX & TPTD & 1.2 & 16.8 & 2/day & 5 \\
\hline 11 & D2M & ovx & TPTD & 6 & 84 & 2/day & 5 \\
\hline 12 & $\mathrm{D} 2 \mathrm{H}$ & ovx & TPTD & 30 & 420 & 2/day & 5 \\
\hline 13 & D3V & ovx & Vehicle & - & - & 3/day & 5 \\
\hline 14 & D3L & OVX & TPTD & 1.2 & 25.2 & 3/day & 5 \\
\hline 15 & D3M & OVX & TPTD & 6 & 126 & 3/day & 5 \\
\hline
\end{tabular}

Abbreviations: TPTD, teriparatide; OVX, ovariectomized. In total, 15 experimental groups were established with four administration schedules: three times a week $(3 / w)$, once a day $(1 / d)$, twice a day $(2 / d)$, and three times a day $(3 / d)$, and four distinct TPTD doses of 0 (vehicle: V), 1.2 (low: $\mathrm{L}$ ), 6 (medium: $\mathrm{M}$ ), and $30 \mu \mathrm{g} \cdot \mathrm{kg}^{-1}$ (high: $\mathrm{H}$ ) for 4 weeks. 


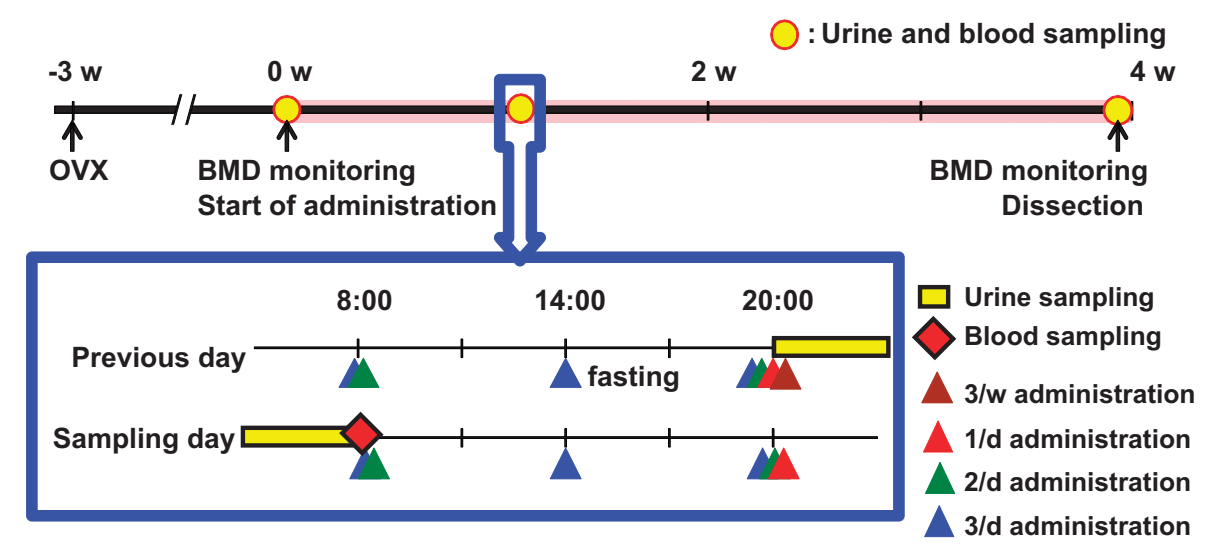

Figure 1. Time course of TPTD administration and sampling. Rats at 13 weeks of age were subjected to either ovariectomy or sham surgery. Three weeks after the surgery, TPTD administration was started. The serum and urine were collected at pre-dosing (day 0), the 9th day (day 9), and the 28th day (day 28) after the initial administration.

(D1 groups) or twice a day (D2 groups); and 1.2 or $6 \mu \mathrm{g} \cdot \mathrm{kg}^{-1}$ of TPTD three times a day (D3 groups) for 4 weeks. Saline injection was given to the sham animals (three times a week) and each group as the vehicle. In total, 15 experimental groups were established and comparatively analyzed.

A double-fluorochrome labeling technique was used to determine the active mineralization sites and rates of bone formation. Calcein (Dojindo Laboratories, Kumamoto, Japan) was subcutaneously injected into each rat twice at a dose of $10 \mathrm{mg} \cdot \mathrm{kg}^{-1}$ body weight on the 8th and 3rd days before killing. This regimen was selected after considering the effects of aging and OVX and to appropriately measure the fluorochrome-based parameters. ${ }^{27}$ After the dosing period, the rats were killed under anesthesia, and the fourth and fifth lumbar vertebrae (LV4 and LV5) were collected. There were no deaths during surgery. The rats awoke from anesthesia $\sim 3 \mathrm{~h}$ after surgery and began feeding. There were no clear behavioral differences after surgery between the OVX- and sham-operated groups. The uterine weight at necropsy revealed uterine atrophy in all rats in the OVX group, thus confirming that the procedure had been performed correctly.

Measurement of bone metabolic markers

The serum and urine were collected at pre-dosing (day 0 ), the 9th day (day 9) and the 28th day (day 28) after the initial administration (Figure 1). All rats were fasted for at least $6 \mathrm{~h}$ before blood and urine collection, and were then placed in metabolic cages without food for $12 \mathrm{~h}$. The serum and urine samples were stored at $-80^{\circ} \mathrm{C}$ until analysis. The time schedules for fasting, urine and blood sampling are also shown in Figure 1.

The urinary level of C-terminal telopeptide of type I collagen (CTX), a bone resorption marker, was measured using a RatLaps EIA (Immunodiagnostic Systems, Boldon, UK). The urinary level of creatinine was measured using L-type Wako CRE.M (Wako Pure Chemical Industries, Osaka, Japan). The urinary level of CTX was corrected for the urinary level of creatinine. The serum level of intact osteocalcin (OC), a bone formation marker, was measured using an OC rat ELISA system (GE Healthcare Bioscience, Tokyo, Japan). All the assays were performed in accordance with the manufacturers' instructions.

Preparation of bone samples

The LV4 was resected at the vertebral arch and the transverse and spinous processes. A central cylindrical specimen with parallel ends and a mean height \pm s.d. of $3.7 \pm 0.1 \mathrm{~mm}$ was obtained from each vertebral body for further measurements by using a diamond band saw (BS-3000; Exakt, Norderstedt, Germany).

The LV5 was removed and dissected free of soft tissue, fixed in $70 \%$ ethanol, stained with Villanueva bone stain, dehydrated in a graded series of ethanol, defatted in acetone and embedded in polymethyl methacrylate (Wako Pure Chemical Industries). Thin sections $(5 \mu \mathrm{m})$ were prepared from a sagittal section of the central LV5. In addition, thin ground sections were prepared from a crosssection of the central LV5 with the remaining blocks.

Measurement of the bone mineral density

The bone mineral density (BMD) of the LV4 vertebral cylinders was measured using dual-energy X-ray absorptiometry (DCS-600EX-3R; Aloka, Tokyo, Japan). The specimens were placed on the scanning table ventral side up and scanned at a pitch of $1 \mathrm{~mm}$ and speed of $25 \mathrm{~mm} \cdot \mathrm{min}^{-1}$. The BMD $\left(\mathrm{mg} \cdot \mathrm{cm}^{-2}\right)$ was calculated from the bone mineral content $(\mathrm{mg})$ and bone area $\left(\mathrm{cm}^{2}\right)$. 
Micro structure analyses for the cortical bone of LV4

A cone-beam X-ray micro-computed tomography (CT) system (ScanXmate-RB090SS150; Comscantecno, Kanagawa, Japan) was used to obtain CT images of the isolated bone samples using the following settings: tube voltage, $70 \mathrm{kV}$; tube current, $0.1 \mathrm{~mA}$; and voxel size, $12.5 \times 12.5 \times 12.5 \mu \mathrm{m}^{3}$. Three-dimensional images were reconstructed and analyzed using the TRI/3D-BON software program (RATOC System Engineering, Tokyo, Japan). We analyzed the middle portion (height, $2 \mathrm{~mm}$; 160 slices) of the lumbar vertebral cylinder. The images were binarized with a fixed threshold (404 $\mathrm{mg} \cdot \mathrm{cm}^{-3}$ converted as BMD). Spatial dissociation of the cortical and trabecular areas was defined by lengths and angles of trabeculae protruding from the inner periosteum according to the manufacturer's instructions.

The cortical bone structure was determined in the ventral cortical bone of LV4 and the following parameters were measured: cortical bone volume $\left(\mathrm{CV}, \mathrm{mm}^{3}\right)$ and cortical void volume ( $\mathrm{V} v / \mathrm{Cv}, \%)$.

Mechanical properties of LV4

Compression testing of the vertebral body was performed as previously described. ${ }^{28}$ The vertebral cylinder specimens of LV4 were placed on a lower platen, cranial-side up and compressed with a 4-mm-diameter upper platen with a material testing machine (EZ-L-1kN; Shimadzu, Tokyo, Japan) at a constant speed of $2 \mathrm{~mm} \cdot \mathrm{min}^{-1}$. The load and displacement curves were recorded, and the following extrinsic parameters were calculated using the testing machine's software program (TRAPEZIUM2; ShimadzU): maximum load $(\mathrm{N})$, stiffness $\left(\mathrm{N} \cdot \mathrm{mm}^{-1}\right)$, and breaking energy (hereafter referred to as energy; $\mathrm{N} \cdot \mathrm{mm}$ ).

Bone histomorphometry and microscopy

The bone histomorphometric parameters related to bone mass, structure, resorption, formation, and turnover were measured using an image analysis system (Histometry RT Camera, System Supply, Nagano, Japan; OsteoMeasure, Decatur, GA, USA). The nomenclature, symbols, and units used in the present study are those recommended by the Nomenclature Committee of the American Society for Bone and Mineral Research. ${ }^{29-30}$ Histomorphometric measurements were obtained from cancellous bone tissue in the secondary spongiosa region of the sagittal sections of LV5.

The following static parameters were measured: trabecular bone volume (BV/TV), trabecular thickness (Tb.Th) and trabecular number (Tb.N). The following bone resorption parameters were measured: eroded surface (ES/BS) and osteoclast surface (Oc.S/BS). The following bone formation and turnover parameters were measured: osteoid surface (OS/BS), mineralizing surface (MS/BS), osteoblast surface (Ob.S/BS, based on the double plus half single label), bone formation rate (BFR/BV), and activation frequency (Ac.f). ${ }^{29-30}$

To analyze the cortical porosity, horizontal sections of LV4 were analyzed using a semi-automated system (OsteoMeasure). The BV/TV, cortical porosity, and osteocyte number (No.Ot/B.Ar) were scored separately from the dorsal and ventral cortical walls and the middle of the trabecular area of the LV4 vertebral body. Small porosities, osteocytic lacunae, and fused lacunae were morphologically dissociated in these scoring in which protrusion and connection of osteocytic canaliculi was the most prominent morphological land mark for this distinction.

Bright-field and fluorescence images of the calceinlabeled undecalcified bone sections were obtained by a microscopy system, ECLIPSE Ni (Nikon, Tokyo, Japan) equipped with differential interference contrast microscopy and objectives (Nikon), as follows: Plan Apo $\lambda \times 10$ (numerical aperture $(\mathrm{NA})=0.45)$, Plan Apo $\lambda \times 20(\mathrm{NA}=$ 0.75), and Plan Apo $\lambda \times 40$ (NA=0.95). The fluorescence signals were obtained using a filter sets, GFP-B (excitation: 460-500 nm, DM: $505 \mathrm{~nm}$, emission: 510-560 nm; Nikon) for calcein and TxRed (excitation: 540-580 nm, DM: $595 \mathrm{~nm}$, emission: 600-660 nm; Nikon) for auto-fluorescence derived from soft tissue. Tiling fluorescence imaging to acquire the entire, high-contrast view of the tissue sections was carried out using a Plan Apo $\lambda \times 10$ objective (NA =0.45). The frame size of a single scan was $1280 \times 1024$ pixels with an 8-bit color depth. The fuorescence and differential interference contrast images were sequentially acquired, with a pixel size of $0.64 \mu \mathrm{m}$. A total of $4 \times 7$ images were combined for tiling. Image processing, including deconvolution, was performed using the imaging software program NIS-elements AR (Nikon).

\section{Statistical analyses}

All the data are presented as mean \pm s.d. The effects of TPTD were investigated using an analysis of variance. The variables showing a difference between the TPTD treatment groups and the vehicle control group of each administration frequency were then analyzed with Dunnett's test (post hoc). Statistical significance was defined as $P<0.05$.

\section{RESULTS}

Effects of TPTD administration regimen in OVX rats on BMD, mechanical properties, and systemic bone markers To analyze the effect of the TPTD administration frequency on bone quality in OVX rats, we established four administration schedules: three times a week (3/w), once a day $(1 / d)$, twice a day $(2 / d)$, and three times a day $(3 / d)$ with 
four distinct TPTD doses: 0 (vehicle: V), 1.2 (low: L), 6 (medium: M), and $30 \mu \mathrm{g} \cdot \mathrm{kg}^{-1}$ (high: H) for 4 weeks (Table 1).

We first analyzed the BMD of LV4 explants by using dual-energy X-ray absorptiometry (Figure 2a). In each group of distinct administration frequency, the BMD was obviously increased in a dose-dependent manner. However, in comparing the groups that received the same TPTD dose at different administration frequencies, we found that an increased administration frequency didn't necessarily augment the BMD. In particular, when the effects on BMD in the $3 / d$ and $2 / d$ groups were compared, the effect in the $3 / d$ group was found to be saturated. An analysis of the association of the average BMD and total amount of TPTD administered ( $\mu \mathrm{g} \cdot \mathrm{kg}^{-1}$ per week; Figure $2 \mathrm{~b}$ ) showed that the BMD increased in a linear manner, especially at total doses $<100 \mu \mathrm{g} \cdot \mathrm{kg}^{-1}$ per week. However, in the groups that received $\geqslant 100 \mu \mathrm{g} \cdot \mathrm{kg}^{-1}$ per week, the effect on BMD appeared to be saturated.

We next analyzed the mechanical properties of the LV4 vertebral body by using the compression tests. As with the $B M D$, the average maximal loads of LV4 increased in a dose-dependent manner (Figure 2c). However, although the increase in the administration frequency for each TPTD dose also effectively augmented the mechanical properties, the effects of the highest frequency of $3 / d$ on these properties were comparable to those in the $2 / \mathrm{d}$ group (Figure 2c). When the average mechanical properties of each regimen were plotted against the total amount of TPTD ( $\mu \mathrm{g} \cdot \mathrm{kg}^{-1}$ per week) (Figure $2 \mathrm{~d}$ ), the effect of TPTD appeared to be saturated at doses $\geqslant 100 \mu \mathrm{g} \cdot \mathrm{kg}^{-1}$ per week.

We also examined the trabecular bone in LV5 by using histomorphological approaches to further evaluate the effect of TPTD on bone metabolism in our regimen settings (Supplementary Figures 1 and 2). As was observed with $B M D, B V / T V$ and Tb.Th were increased in a dosedependent manner in each frequency group, and these parameters in the 3/d group were comparable to those in the 2/d group (Supplementary Figure 1A and B), although Tb.N did not show significant differences among any of the regimens (Supplementary Figure 1C). Bone formation parameters such as OS/BS, MS/BS, and Ob.S/BS showed similar tendencies to BV/TV and Tb.Th (Supplementary Figure 1D-F). Of note, $30 \mu \mathrm{g} \cdot \mathrm{kg}^{-1}$ at $2 / \mathrm{d}(\mathrm{H}: 2 / \mathrm{d})$ did not markedly augment these parameters compared to $6 \mu \mathrm{g} \cdot \mathrm{kg}^{-1}$ at 2/d (M: 2/d), indicating the saturation of the effects of TPTD administration on bone formation.

The bone resorption parameters such as ES/BS and Oc.S/BS did not show significant differences from the control group in any of the regimens; however, $1.2 \mu \mathrm{g} \cdot \mathrm{kg}^{-1}$ at $3 / \mathrm{d}(\mathrm{L}: 3 / \mathrm{d})$ and $6 \mu \mathrm{g} \cdot \mathrm{kg}^{-1}$ at $3 / \mathrm{d}(\mathrm{M}: 3 / \mathrm{d})$ augmented these bone resorption parameters, albeit not significantly
(Supplementary Figure 2A and B). As with other turnover parameters, BFR/BV was significantly augmented by TPTD administration regimens, except for $6 \mu \mathrm{g} \cdot \mathrm{kg}^{-1}$ at 3/w (M: 3/ W) and $1.2 \mu \mathrm{g} \cdot \mathrm{kg}^{-1}$ at 1/d (L: 1/d), whereas Ac.f exhibited similar patterns to the bone formation parameters (Supplementary Figure 2C and D).

To investigate the effects on systemic bone turnover, the changes in the levels of serum OC and urine CTX were examined as markers of bone formation and resorption, respectively (Figure 2e, f and Supplementary Figure 3A-D). The change in the rates of the average serum OC level were markedly increased compared to that of the vehicle control in each frequency group in a dose-dependent manner, although significant changes were observed only for $6 \mu \mathrm{g} \cdot \mathrm{kg}^{-1}$ at 2/d (M: 2/D), $30 \mu \mathrm{g} \cdot \mathrm{kg}^{-1}$ at $2 / \mathrm{d}$ $(\mathrm{H}: 2 / \mathrm{D})$, and $1.2 \mu \mathrm{g} \cdot \mathrm{kg}^{-1}$ at $3 / \mathrm{d}$ (M: 3/d; Figure 2e). Furthermore, when the same doses of TPTD in the distinct frequency groups were compared, the changes in the $\mathrm{OC}$ levels also increased in a frequency-dependent manner (Figure 2e).

The effects of the TPTD regimens on the average urine CTX showed distinct patterns from those on the average serum $\mathrm{OC}$ levels (Figure $2 \mathrm{f}$ and Supplementary Figure 3E$\mathrm{H})$. At the lowest dose of $1.2 \mu \mathrm{g} \cdot \mathrm{kg}^{-1}$, only the CTX level in the highest frequency of $3 / d$ ( $L: 3 / d$ ) was increased, albeit not significantly; in contrast, in the other frequency groups, the effects of $1.2 \mu \mathrm{g} \cdot \mathrm{kg}^{-1}$ of TPTD administration (L: 1/d and L: 2/d) on the CTX level were comparable to those of the vehicle controls, demonstrating an obvious contrast in the effects of the different regimens on the OC levels. Significant increases in the CTX levels by TPTD administration were observed only in groups receiving $30 \mu \mathrm{g} \cdot \mathrm{kg}^{-1}$ at 2/d (H: 2/D) and $1.2 \mu \mathrm{g} \cdot \mathrm{kg}^{-1}$ at $3 / \mathrm{d}(\mathrm{M}: 3 / \mathrm{d})$. Therefore, the augmented effect on the CTX level by TPTD is only achieved at a higher administration frequency and higher dose than those of the effects on the OC level. Serum Ca levels in any settings did not show significant differences (data not shown).

Spatial revelation of the development of cortical porosity depending on the frequency and dose of TPTD administration

We next assessed whether the administration frequency and/or dose of TPTD affects the cortical porosity, via microCT-based analyses. Whole-body scanning of specimens revealed that the vertebral bodies exhibited the most obvious cortical porosities in some regimen groups. We therefore focused our analyses on the lumbar vertebrae. Figure 4 shows three-dimensional reconstitution images of micro-CT scanning of LV4. Ventral views of a representative specimen in each regimen are arranged with the cortical porosity distribution area highlighted in blue. We observed 
a

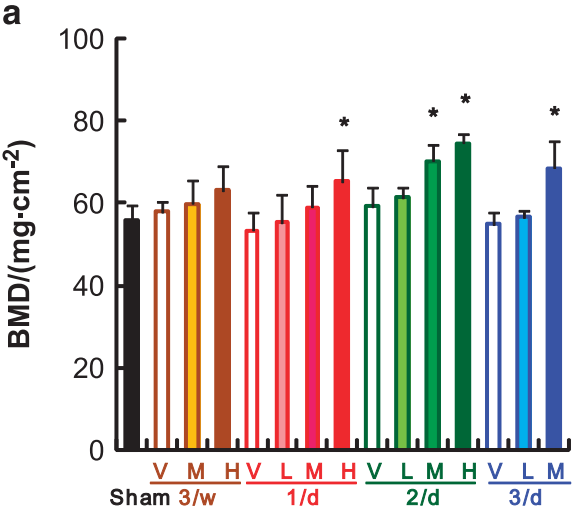

C
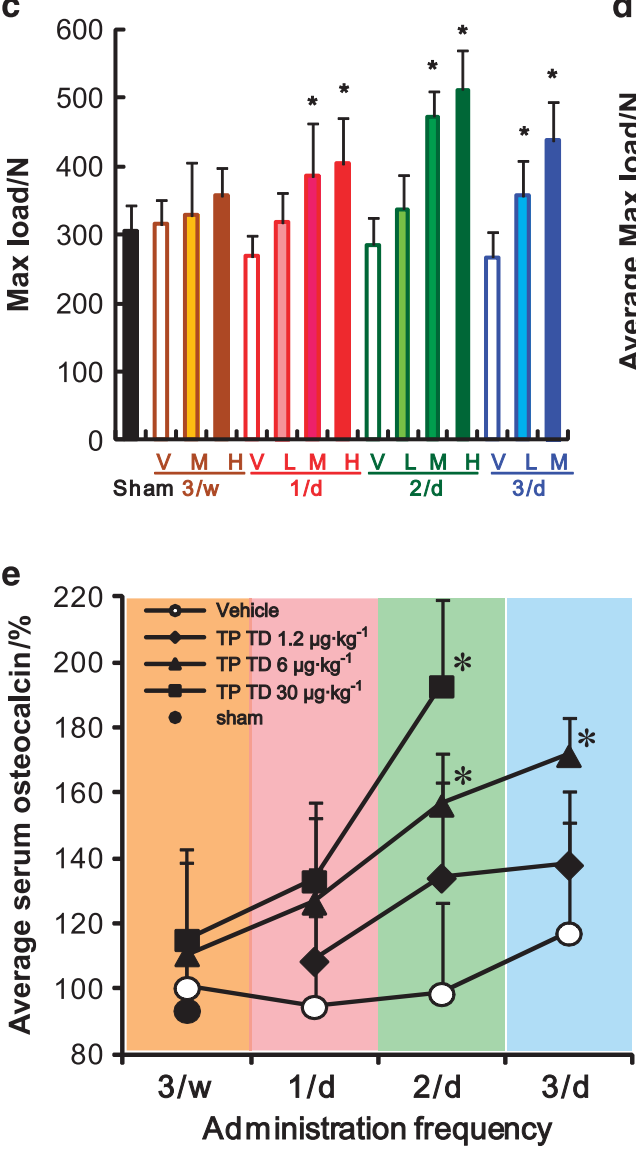

d

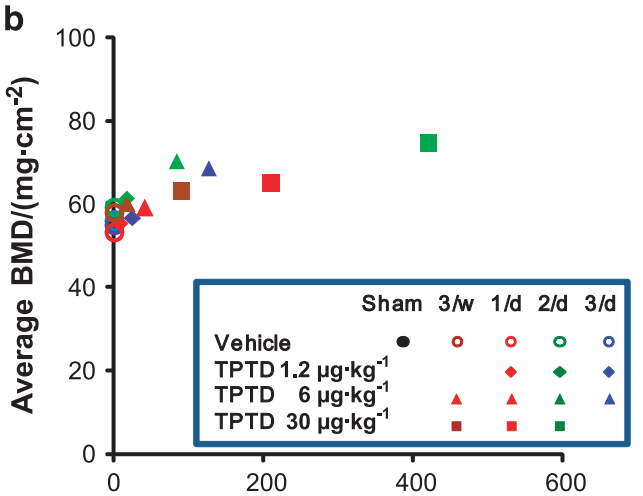

Total amount of TPTD/( $\mu \mathrm{g} \cdot \mathrm{kg}^{-1}$ per week)

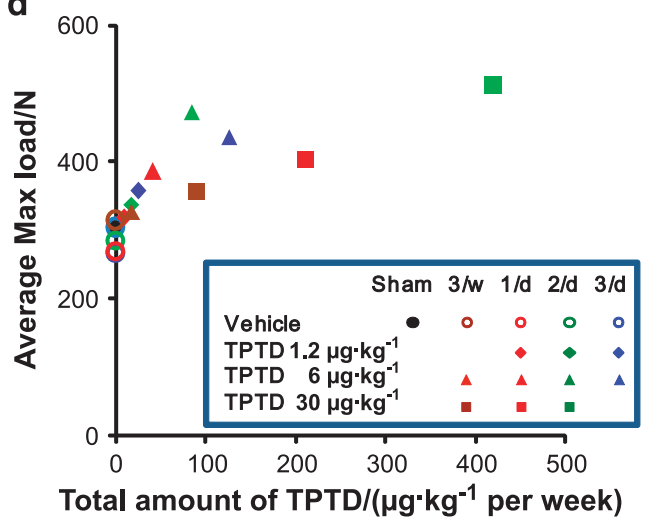

f

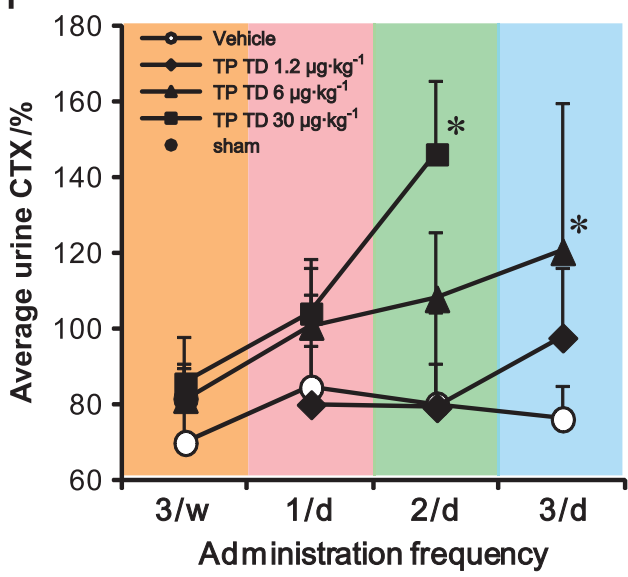

Figure 2. The bone mineral density and mechanical strength of the 4th lumbar vertebral body, and changes in the bone metabolic markers in serum and urine. $(\mathbf{a}, \mathbf{b})$ BMD of LV4 vertebral body measured by dual-energy X-ray absorptiometry is plotted against the administration regimen; (a) and total amount of TPTD administered $\left(\mu \mathrm{g} \cdot \mathrm{kg}^{-1}\right.$ per week; $\left.\mathbf{b}\right)$. (c, d) The mechanical properties of the LV4 vertebral body as measured by a compression test are plotted against the administration regimen (c) and the total amount of TPTD $\left(\mu \mathrm{g} \cdot \mathrm{kg}^{-1}\right.$ per week) administered (d). (a and c) The data are shown as the means+s.d. $\left(n=4\right.$ or 5). ${ }^{*}$ Indicates $P<0.05$ vs vehicle of each administration frequency (analysis of variance (ANOVA) with post hoc Dunnett's tests). V: vehicle control, L: $1.2 \mu \mathrm{g} \cdot \mathrm{kg}^{-1}, \mathrm{M}: 6 \mu \mathrm{g} \cdot \mathrm{kg}^{-1}, \mathrm{H}: 30 \mu \mathrm{g} \cdot \mathrm{kg}^{-1}$. (b, d) The data are shown as the means ( $\left.n=4 \mathrm{or} 5\right)$. Black closed circle: Sham control. Blue: three times a day, green: two times a day, red: once a day, brown: three times a week. Opened circle: vehicle control, diamond: $1.2 \mu \mathrm{g} \cdot \mathrm{kg}^{-1}$, triangle: $6 \mu \mathrm{g} \cdot \mathrm{kg}^{-1}$, square: $30 \mu \mathrm{g} \cdot \mathrm{kg}^{-1} \cdot(\mathbf{e}, \mathbf{f})$ Serum osteocalcin level (e) and urine CTX level (f) at day 9 are plotted against the administration frequency of TPTD. (e, f) The data are shown as the means $(n=4$ or 5$) .{ }^{*}$ Indicates $P<0.05$ vs vehicle of each administration frequency (two-way repeated measure ANOVA with post hoc Dunnett's test). Black closed circle: Sham control, opened circle: vehicle control, diamond: $1.2 \mu \mathrm{g} \cdot \mathrm{kg}^{-1}$, triangle: $6 \mu \mathrm{g} \cdot \mathrm{kg}^{-1}$, square: $30 \mu \mathrm{g} \cdot \mathrm{kg}^{-1}$. 
increases in the distributions of cortical porosity, particularly in the ventral cortical bone, in the regimen groups of $30 \mu \mathrm{g} \cdot \mathrm{kg}^{-1}$ at $2 / \mathrm{d}(\mathrm{H}: 2 / \mathrm{d})$ and $6 \mu \mathrm{g} \cdot \mathrm{kg}^{-1}$ at $3 / \mathrm{d}(\mathrm{M}: 3 / \mathrm{d}$; Figure 3a). Significant increases in the cortical volume (Cv) were observed at $30 \mu \mathrm{g} \cdot \mathrm{kg}^{-1}$ in the $1 / \mathrm{d}$ and $2 / \mathrm{d}$ groups $(\mathrm{H}$ : $1 / \mathrm{d}$ and $\mathrm{H}: 2 / \mathrm{d}$; Figure $3 \mathrm{~b}$ ). In contrast, significant increases in the void volume/cortical volume ( $\mathrm{Vv} / \mathrm{CV}$ ) were observed in the groups receiving $30 \mu \mathrm{g} \cdot \mathrm{kg}^{-1}$ at $2 / \mathrm{d}(\mathrm{H}: 2 / \mathrm{d})$ and $6 \mu \mathrm{g} \cdot \mathrm{kg}^{-1}$ at $3 / \mathrm{d}(\mathrm{M}: 3 / \mathrm{d})$, suggesting that the appearance of cortical porosity is sensitive to the administration frequency as well as the total dose of TPTD (Figure 3c).

We next applied histomorphometric analyses to further analyze the cortical porosity caused by TPTD administration (Figure 4). Since our micro-CT-based observations indicated that cortical porosity was preferentially observed in the ventral cortical portion of LV4, the dorsal and ventral cortical bone were analyzed and compared. Histomorphometric analyses of the ventral cortical bone revealed obvious increases in the average cortical porosity in the regimens of $30 \mu \mathrm{g} \cdot \mathrm{kg}^{-1}$ at $2 / \mathrm{d}(\mathrm{H}: 2 / \mathrm{d})$ and $6 \mu \mathrm{g} \cdot \mathrm{kg}^{-1}$ at $3 / \mathrm{d}$ (M: 3/d; Figure $4 a$ ).

We next plotted the average cortical porosity (\%) against the total amount of TPTD administration $\left(\mu \mathrm{g} \cdot \mathrm{kg}^{-1}\right.$ per week; Figure $4 \mathrm{~b})$. In the ventral cortical potion, a marked increase in the cortical porosity (>15\%) was observed in the regimens of $30 \mu \mathrm{g} \cdot \mathrm{kg}^{-1}$ at $2 / \mathrm{d}(\mathrm{H}: 2 / \mathrm{d})$ and $6 \mu \mathrm{g} \cdot \mathrm{kg}^{-1}$ at $3 / \mathrm{d}(\mathrm{M}: 3 / \mathrm{d})$, whereas other regimens showed increases of $<10 \%$. For the dorsal cortical portion,
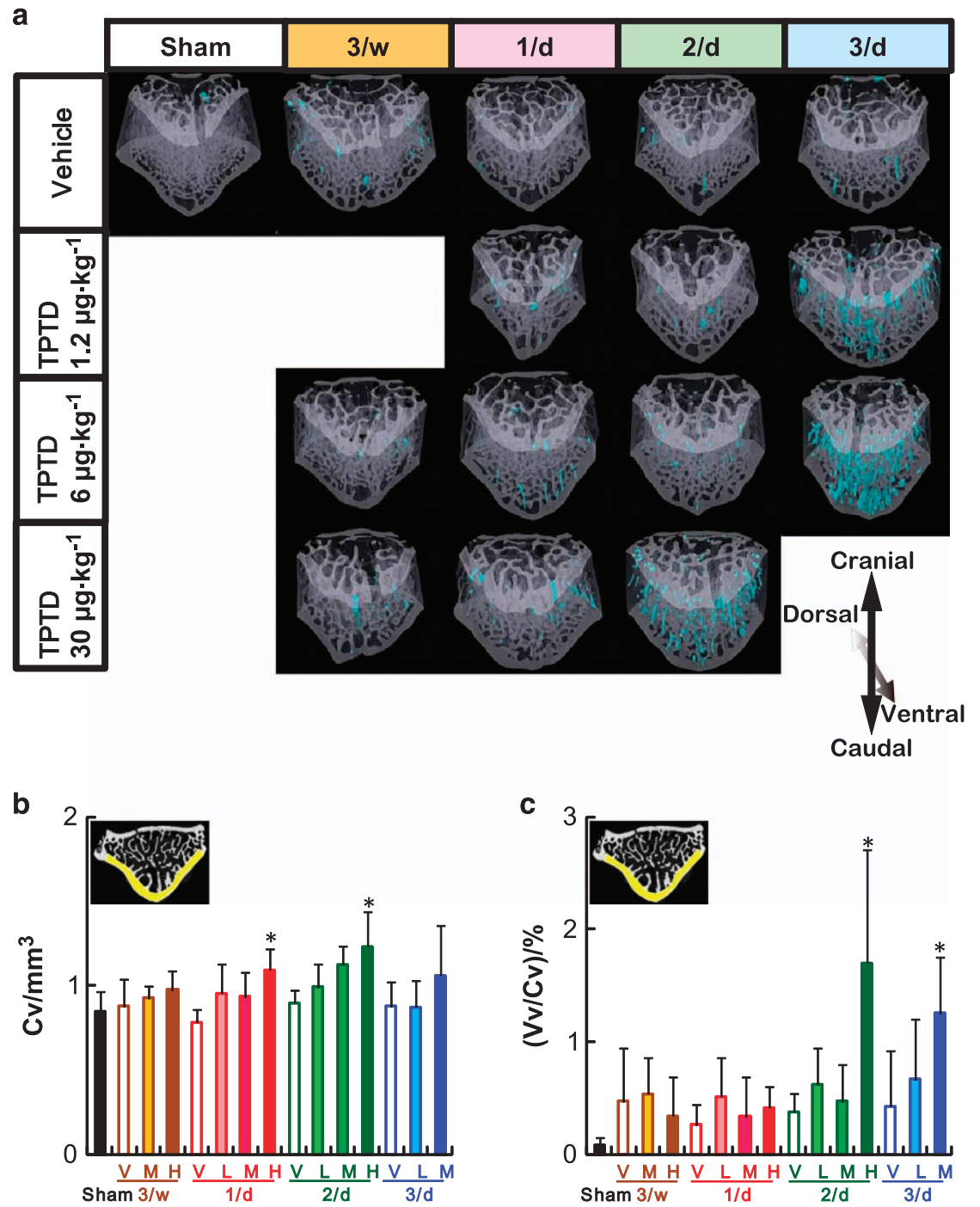

Figure 3. A micro-CT analysis of the cortical bone of LV4. (a) The three-dimensional reconstitution images of the bone structure of LV4. The region of cortical porosity is highlighted in blue. $(\mathbf{b}, \mathbf{c})$ Cortical bone volume $(\mathrm{Cv})$ and $(\mathbf{b})$ cortical void volume $(\mathrm{Vv} / \mathrm{Cv})(\mathbf{c})$ of ventral cortical bone of LV4 are plotted against the administration regimen of TPTD. The data are shown as the means+s.d. $(n=4$ or 5$) .{ }^{*}$ Indicates $P<0.05$ vs vehicle of each administration frequency (analysis of variance with post hoc Dunnett's test). V: vehicle control, L: $1.2 \mu \mathrm{g} \cdot \mathrm{kg}^{-1}, \mathrm{M}^{4} 6 \mu \mathrm{g} \cdot \mathrm{kg}^{-1}, \mathrm{H}: 30 \mu \mathrm{g} \cdot \mathrm{kg}^{-1}$. 

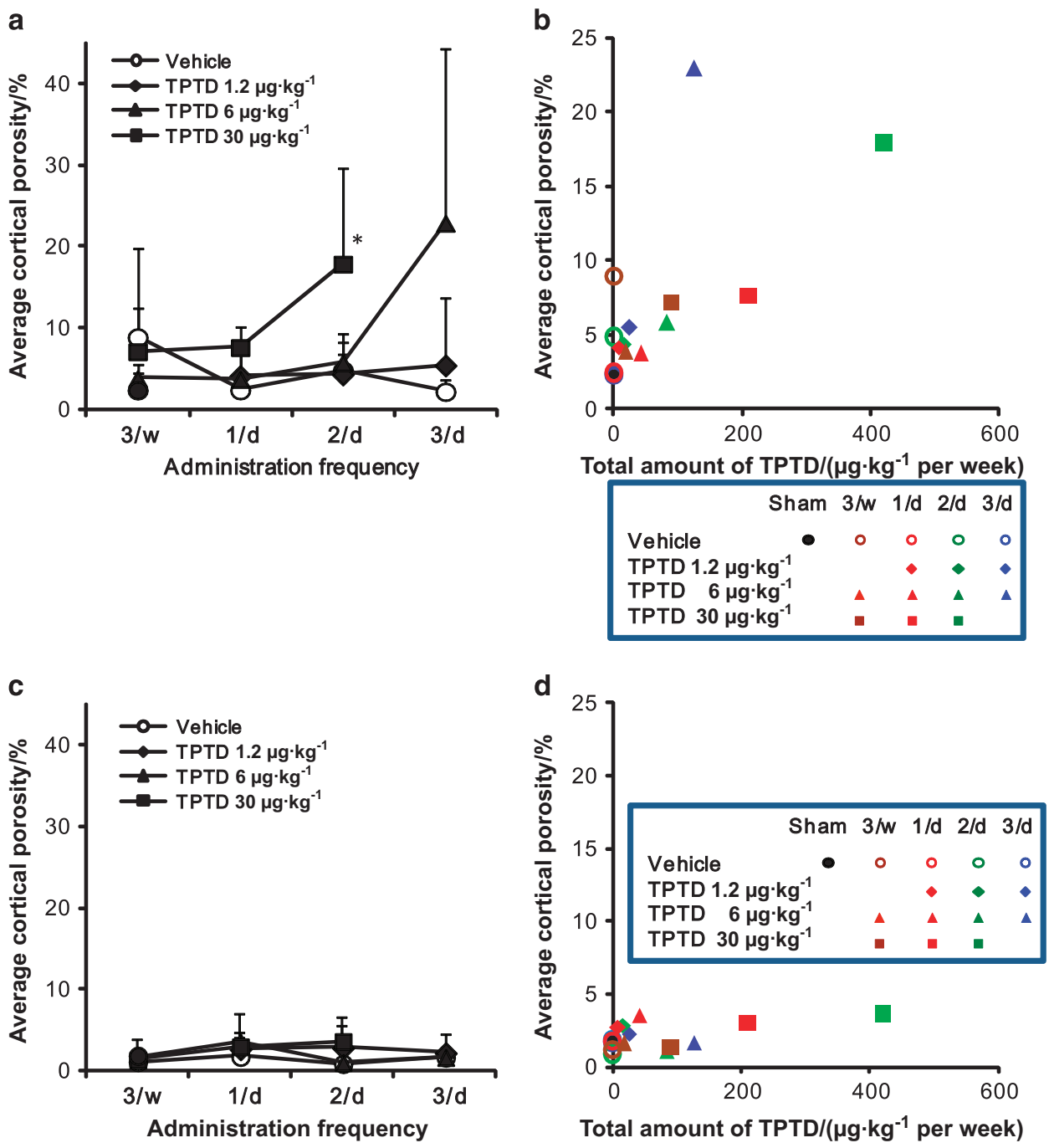

Figure 4. A comparison of the average cortical porosity between the ventral and dorsal portions of LV4. (a, b) The average cortical porosity in ventral wall of LV4 is plotted against the administration frequency (A) and total amount of TPTD administered ( $\mu \mathrm{g} \cdot \mathrm{kg}^{-1}$ per week; $\left.\mathbf{b}\right)$. (c, d) The average cortical porosity in the dorsal wall of LV4 is plotted against the administration frequency (c) and total amount of TPTD administered $\left(\mu \mathrm{g} \cdot \mathrm{kg}^{-1}\right.$ per week; d). (a, c) The data are shown as the means ( $n=4$ or 5). Black closed circle: Sham control, opened circle: vehicle control, diamond: $1.2 \mu \mathrm{g} \cdot \mathrm{kg}^{-1}$, triangle: $6 \mu \mathrm{g} \cdot \mathrm{kg}^{-1}$, square: $30 \mu \mathrm{g} \cdot \mathrm{kg}^{-1}$. (b, d) Data represent means $(n=4$ or 5). Black closed circle: Sham control. Blue: three times a day, green: two times a day, red: once a day, brown: three times a week. Opened circle: vehicle control, diamond: $1.2 \mu \mathrm{g} \cdot \mathrm{kg}^{-1}$, triangle: $6 \mu \mathrm{g} \cdot \mathrm{kg}^{-1}$, square: $30 \mu \mathrm{g} \cdot \mathrm{kg}^{-1}$.

all of the regimens showed scores of $<5 \%$ (Figure $4 \mathrm{C}$ and d). These findings suggested that the augmentation of the cortical porosity was due not merely to changes in the total dose of TPTD but also the frequency of administration. This was specifically demonstrated by the fact that, although the total amount of TPTD administered in the regimen of $30 \mu \mathrm{g} \cdot \mathrm{kg}^{-1}$ at 1 day ( $\left.\mathrm{H}: 1 / \mathrm{d}\right)$ was higher than that in the regimen of $6 \mu \mathrm{g} \cdot \mathrm{kg}^{-1}$ at 3 days (M: $\left.3 / \mathrm{d}\right)$, the porosity score was $<10 \%$ when compared with the vehicle controls. Furthermore, this phenomenon was demonstrated to be highly site-specific in the same bone elements of the vertebral body.
Ventral wall-specific bone outgrowth and development of cortical porosity demonstrated by tiling fluorescence imaging

To assess the site-specific distinction described above, we used fluorescence tiling imaging with a deconvolution fluorescence microscopy, which enables the depiction of the spatial distribution of fluorescence signals in a whole-tissue section ${ }^{31}$ (Figure 5). Figure 5 shows the fluorescence tiling images of LV4 for the sham vehicle control, OVX vehicle control ( $\mathrm{V}: 3 / \mathrm{d}), 30 \mu \mathrm{g} \cdot \mathrm{kg}^{-1}$ at 1 day $(\mathrm{H}: 1 / \mathrm{d}), 30 \mu \mathrm{g} \cdot \mathrm{kg}^{-1}$ at 2 days $(\mathrm{H}: 2 / \mathrm{d})$, and $6 \mu \mathrm{g} \cdot \mathrm{kg}^{-1}$ at 3 days (M: $3 / d$ ) groups (Figure $5 a-e$, respectively). Green 
a

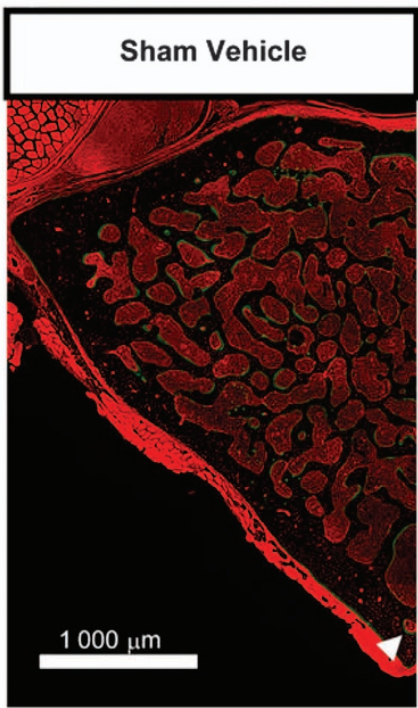

b

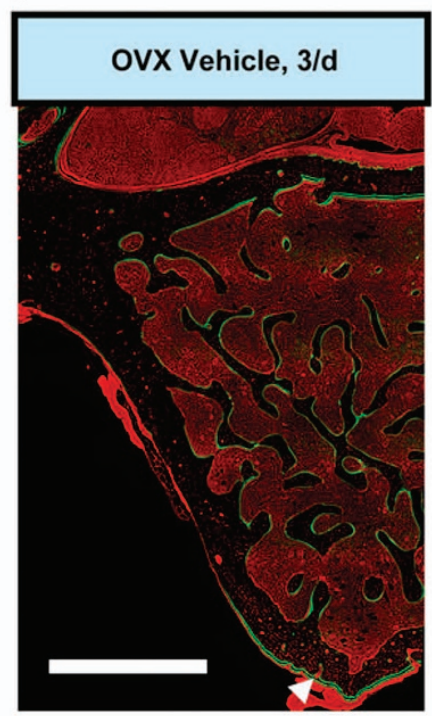

c

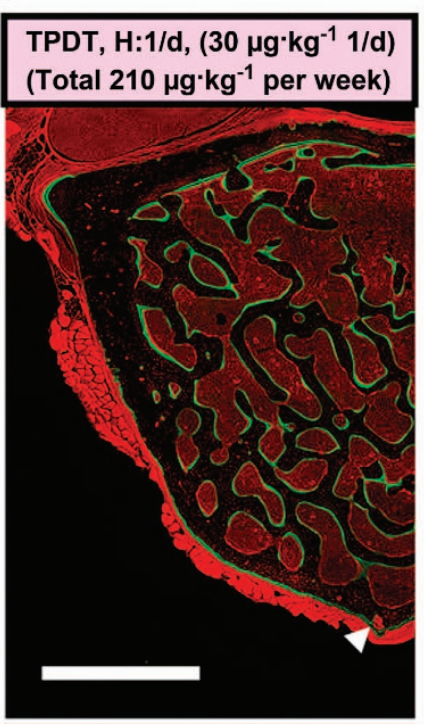

d

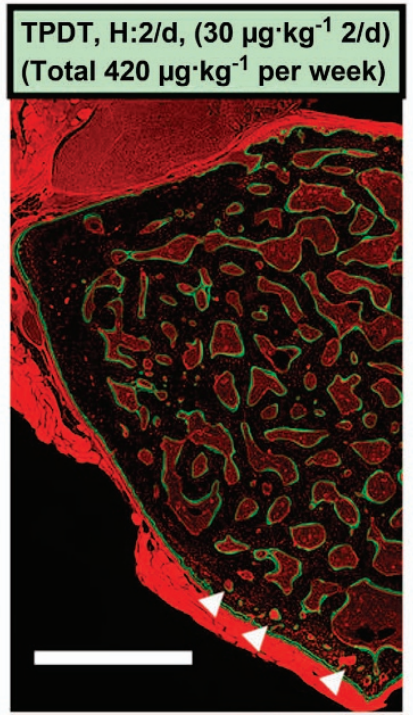

e

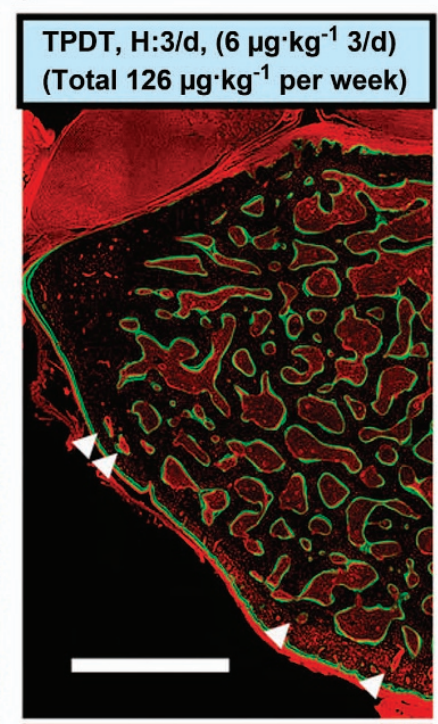

Figure 5. Fluorescence tiling images with deconvolution fluorescence microscopy of transverse sections of the vertebral bodies obtained from specimens in the sham vehicle group, OVX vehicle group (V: 3/d), and $30 \mu \mathrm{g} \cdot \mathrm{kg}^{-1}$ at 1 day (H: $\left.1 / \mathrm{d}\right), 30 \mu \mathrm{g} \cdot \mathrm{kg}^{-1}$ at $2 \mathrm{days}(\mathrm{H}: 2 / \mathrm{d})$, and $6 \mu \mathrm{g} \cdot \mathrm{kg}^{-1}$ at 3 days (M: 3/d) groups (a-e, respectively). The green fluorescence signal from calcein labeling demarcates active bone formation sites, whereas the auto-fluorescence signal derived from soft tissue provides morphological information. The white arrowheads indicate obvious intracortical bone resorption. Scale bars, $1000 \mu \mathrm{m}$. Dorsal to up. The corresponding bright-field images with differential interference contrast (DIC) are shown in Supplementary Figure 4.

fluorescence signals with calcein labeling showed active bone formation in the trabecular bone and periosteal region of the ventral cortical wall, but not in the dorsal wall, at all TPTD administration levels, indicating the stimulated ventral outgrowth of the vertebral body. Red fluorescence signals by the auto-fluorescence of the soft tissue, such as muscle, periosteum, bone marrow, bone cavities encircling blood vessels, and osteocytic lacunae, clearly demarcated the spatial distribution of the unmineralized tissues. Consistent with the observations on micro-CT, the development of cortical porosity due to intracortical bone resorption was observed predominantly in the ventral cortical walls in specimens from the $30 \mu \mathrm{g} \cdot \mathrm{kg}^{-1}$ at 2 days ( $\mathrm{H}: 2 / \mathrm{d}$ ) and $6 \mu \mathrm{g} \cdot \mathrm{kg}^{-1}$ at 3 day (M: $3 / \mathrm{d}$ ) groups (shown by white arrowheads). In these regimens, we further noticed a greater accumulation of red fluorescence signals derived from osteocytic lacunae in the ventral cortical wall in the dorsal wall, which was also confirmed by differential interference contrast tiling images of the corresponding specimens (Supplementary Figure 4). 
10

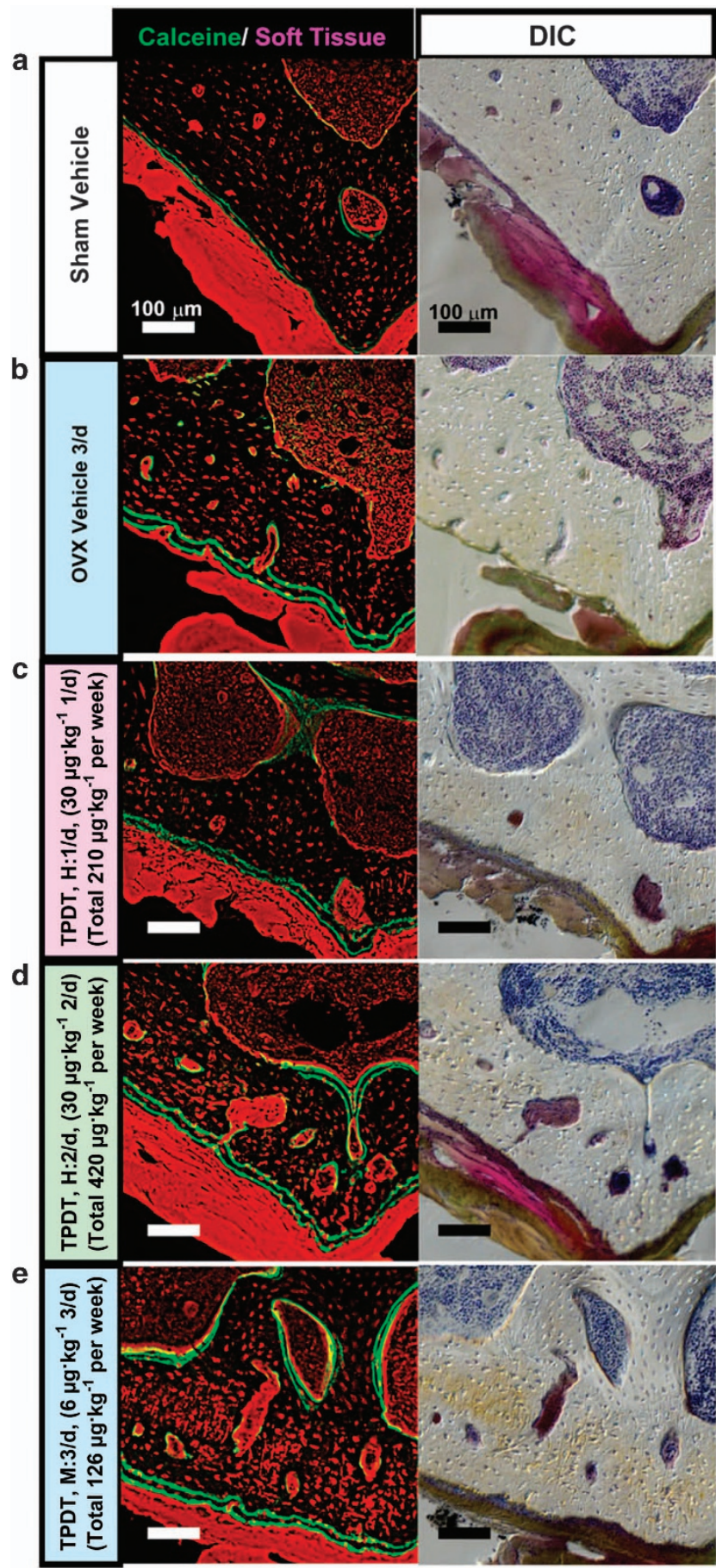

Figure 6. Magnified views focusing on the ventral portion of the large tiling images shown in Figure 5. The images acquired from specimens of the sham vehicle group, OVX vehicle group (V:3/d), and $30 \mu \mathrm{g} \cdot \mathrm{kg}^{-1}$ at 1 day (H: $1 / \mathrm{d}), 30 \mu \mathrm{g} \cdot \mathrm{kg}^{-1}$ at 2 days (H: $\left.2 / \mathrm{d}\right)$, and $6 \mu \mathrm{g} \cdot \mathrm{kg}^{-1}$ at 3 days (M: 3/d) groups (a-e, respectively) are arranged with corresponding bright-field images with differential interference contrast (DIC). Scale bar, $100 \mu \mathrm{m}$. Dorsal to up.
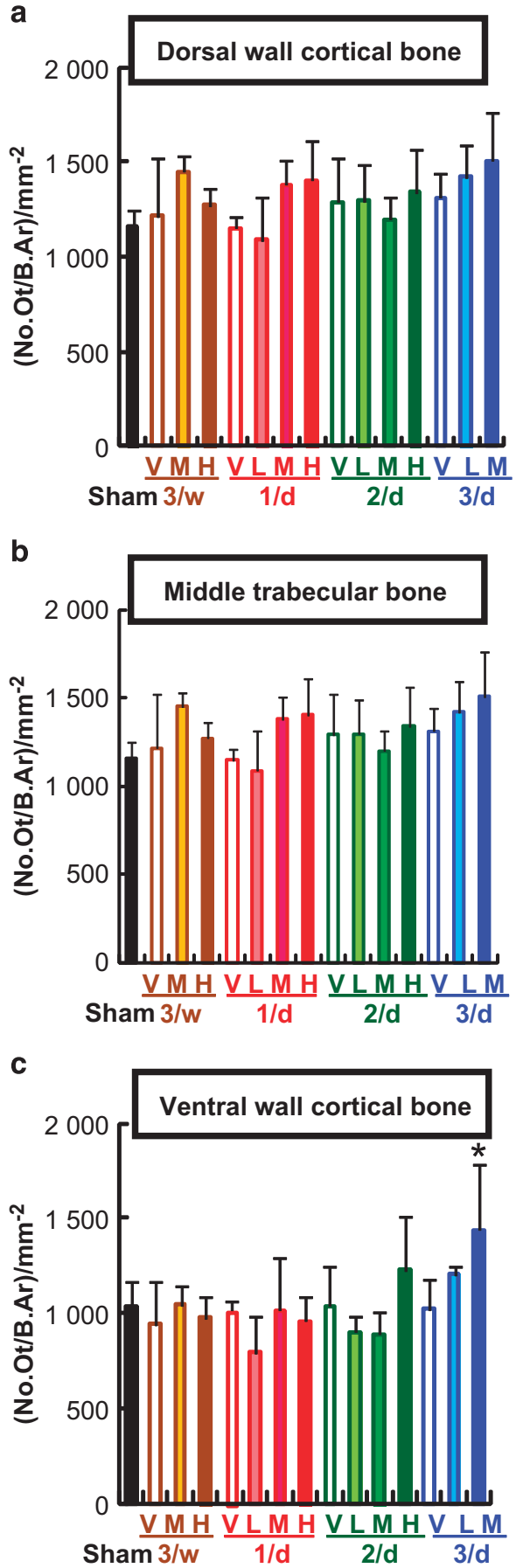

Figure 7. A comparison of the number of osteocytes. A histomorphometric analysis of the sagittal sections of the LV4 $(\mathbf{a}-\mathbf{c})$ parameters of dorsal cortical wall (a), middle trabecular area (b), and ventral cortical wall (c) are shown. The data are shown as the means+s.d. $(n=4$ or 5$)$. *Indicates $P<0.05$ vs vehicle of each administration frequency (analysis of variance with post hoc Dunnett's test). V: vehicle control, L: $1.2 \mu \mathrm{g} \cdot \mathrm{kg}^{-1}, \mathrm{M}: 6 \mu \mathrm{g} \cdot \mathrm{kg}^{-1}, \mathrm{H}$ : $30 \mu \mathrm{g} \cdot \mathrm{kg}^{-1}$. 
Contribution of the increased number of osteocytes to cortical porosity

To further assess these distinct spatial distribution patterns of osteocytic lacunae, we observed the same specimens with zoomed views of the tiling images, focusing on the ventral cortical walls (Figure 6). In specimens from the $30 \mu \mathrm{g} \cdot \mathrm{kg}^{-1}$ at 2 days ( $\mathrm{H}: 2 / \mathrm{d}$ ) and $6 \mu \mathrm{g} \cdot \mathrm{kg}^{-1}$ at 3 day (M: $3 / \mathrm{d}$ ) groups, an anisotropic arrangement and increased size and number of osteocytic lacunae were obviously demonstrated. Of note, some of the osteocytic lacunae were fused, thus contributing to the cortical void formation.

A histomorphometrical analysis demonstrated that the ventral cortical wall in specimens receiving these regimens had higher numbers of osteocytes than those in specimens from other regimens (Figure 7). Therefore, the fused lacunae might have developed due to the increased proximity of osteocytic lacunae induced by overdose or high-frequency administration of TPTD.

\section{DISCUSSION}

This study used a standard OVX rat model of postmenopausal osteoporosis to examine the effects of TPTD at distinct dosage and frequency of administration on $B M D$, mechanical properties of bone, systemic bone formation and resorption markers, and development of cortical porosity. In our regimens, doses of TPTD administered to rats were determined by pharmaco-kinetic comparisons between rats and post-menopausal women, whereas administration schedule was established by comparative analyses of the bone turnover rate of OVX rats and post-menopausal women. ${ }^{27,32-35}$ As a common $O V X$ rat model to examine the pharmacological action of TPTD, we performed OVX surgery in animals at 13 weeks of age. ${ }^{23-25}$ In this model, a significant reduction in the BMD in the OVX group compared with that in the sham group is observed starting from 3 months after the surgery. ${ }^{23,36-39}$ This study monitored blood and urine samples until 7 weeks after surgery ( 4 weeks after the initial TPTD treatment), and dissected specimens at 7 weeks after the surgery for morphological evaluations to observe the early pharmacological actions of TPTD, especially on changes in the microarchitecture. In fact, we did not observe significant differences in the BMD, mechanical properties, average serum OC, or average cortical porosity between the sham group and OVX vehicle group, which is consistent with our previous report. ${ }^{27}$ Nevertheless, our analyses demonstrated that the effects of TPTD on the BMD, mechanical properties, and bone turnover were fundamentally associated with the total amount of TPTD administered, whereas the development of cortical porosity was affected by the administration frequency as well as the dosage.
Under our established regimens, the groups of $30 \mu \mathrm{g} \cdot \mathrm{kg}^{-1}$ at 2 days (H:2/d) and $6 \mu \mathrm{g} \cdot \mathrm{kg}^{-1}$ at 3 day (M: $3 / \mathrm{d}$ ) showed marked increase in the cortical porosity in the ventral portion of the vertebral body. These regimen groups also showed significant increases in the serum level of CTX as a marker of bone resorption. This correlation suggests that the development of cortical porosity strongly contributes to the systemic bone resorption marker levels in this experimental model study. Supporting this notion was our observation that the $30 \mu \mathrm{g} \cdot \mathrm{kg}^{-1}$ at 2 days (H: $\left.2 / \mathrm{d}\right)$ and $6 \mu \mathrm{g} \cdot \mathrm{kg}^{-1}$ at 3 day (M: $3 / \mathrm{d}$ ) groups did not have significantly augmented ES/BS or Oc.S/BS in the trabecular bone. This finding in our rat OVX model is consistent with those from a clinical study in elderly post-menopausal women in which increased levels of bone turnover makers were associated with higher cortical porosity and thinner cortices. ${ }^{17}$

The increased trabecular BMD and the development of intracortical bone resorption mentioned above phenocopies transgenic mice expressing the constitutive-active form of PTHIR (CAPTHIR) driven by osteoblast- or osteocyte-specific promoters such as collal and Dmpl, respectively. ${ }^{40-41}$ Therefore, high-frequency administration as well as over-dosage of TPTD may exert similar effects on bone properties to that of constitutively activated PTHIRmediated signaling in osteoblast-osteocyte lineages.

The development of cortical porosity in these regimens was preferentially observed in the ventral cortical wall of vertebrae, suggesting that the local bone environment and/or site-specific bone architecture could be a critical rationale for developing cortical porosity, as well as the regimen of TPTD administration. Deconvolution fluorescence tiling imaging demonstrated that TPTD administration preferentially stimulated the outgrowth of the ventral cortical bone, in which the development of cortical porosity was also predominantly observed. As the anabolic effect of TPTD administration is reportedly associated with mechanical loading to the bone, ${ }^{42-43}$ local distinctions in mechanical loading are likely to affect the site-specific bone growth and the development of intracortical bone resorption.

Our findings further showed that the increased number and proximity of osteocytic lacunae occasionally forming fused lacunae induced by TPTD can be a critical element involved in developing cortical porosity. Several studies have suggested that distinct mechanical loading at local bone sites affects the microarchitecture and functions of osteocyte-mediated cellular networks, thereby contributing to the locally different properties of bone remodeling that respond to local and systemic regulations. ${ }^{44-45}$ Therefore, site-specific increases in the osteocytic lacunae induced by TPTD administration may also be related to local distinctions in mechanical loading. 
The molecules that regulate the transition from osteoblasts to osteocytes are largely unknown. ${ }^{46}$ In addition, how the ratio of this transition from a given population of bone-forming osteoblasts is controlled is also unknown. Morphologically, the transition to osteocytes involves dynamic changes in cellular shape, such as the extension of the cellular process and reduction in cell body size. ${ }^{47-48}$ Generally, the reduction in cell size is tightly associated with the reduction in cytoplasmic organelles such as mitochondria and the endoplasmic reticulum. ${ }^{49}$ Therefore, the local vasculature that supplies oxygen to the adjacent cells may affect the rate of osteocyte differentiation as well as osteocyte function. However, how the mechanical loading and stimulated rate of bone formation by TPTD administration affect the formation rate of osteocytes remains to be clarified.

The increased size of the osteocytic lacunae observed in bone sites that develop cortical porosity suggests the involvement of osteocytic osteolysis in forming the fused lacunae and cortical porosity. ${ }^{50-51}$ Qing et al ${ }^{52}$ reported that tartrate-resistant acid phosphatase and cathepsin K were involved in osteocytic osteolysis. They also reported that no osteocytic remodeling was observed with experimental unloading. These present and previous findings suggest that bone site-specific distinction in mechanical loading may be a critical factor for the induction of osteocytic osteolysis, which could contribute to the development of intracortical porosity.

PTH binding to PTHIR preferentially simulates endosomemediated long CAMP signaling, whereas PTHrP prefers to stimulate canonical short CAMP signaling. ${ }^{9}$ The stabilized PTH analog D6 exhibits a longer half-life than native PTH(1-34) in the blood stream, inducing an increase in the blood $\mathrm{Ca}^{2+}$ level that persists longer than that of PTH(1-34). ${ }^{10}$ This report indicates that a prolonged increase in the blood PTH level is closely associated with the catabolic effect of PTH. The other PTH analog, M-PTH(1-34), selectively binds to the RO state PTHIR which preferentially stimulates the endosome-mediated long signal. ${ }^{11}$ The half-life of this analog in the blood stream is much shorter than that of PTH(1-34). However, it stimulates a longer calcemic response than does PTH(1-34), which is similar to the effects of D6. This observation is explained by the fact that M-PTH(1-34) binds to a specific PTHIR conformation (the RO state) in a pseudo-irreversible manner. These findings together demonstrate that continuous or prolonged stimulation of the endosome-mediated long CAMP signaling by PTH exerts a catabolic effect on bone metabolism.

On the basis of our series of comparative studies of pharmaco-dynamics and bone turnover rate between OVX rats and post-menopausal women, the single administration dose of $6 \mu \mathrm{g} \cdot \mathrm{kg}^{-1}$ used in this study corresponds to a dose two times higher than that of $56.5 \mu \mathrm{g}$ once-weekly administration used in clinical setting based on the areas under the concentration-time curves, ${ }^{53-55}$ whereas once daily and three times weekly administrations in rats are estimated to be equivalent to once-weekly administration in post-menopausal women. ${ }^{27,32-35}$ Therefore, our observation in this work suggests that over-frequency or over-doses of TPTD administration in clinical situations may be a causative of cortical porosity, whose development is associated with local distinction in mechanical properties of bone sites. Although these findings were obtained from experiments with young adult rats, the same regimens may also cause cortical porosity in older rats, as our previous study has demonstrated the effects of TPTD in aged OVX rats. ${ }^{24}$ Given that clinical evidence has indicated the relevance of cortical porosity and thinner cortices as determinants of bone fragility and fracture risk, ${ }^{15-18}$ our findings here underscore the importance of the TPTD treatment regimen in inhibiting the development of cortical porosity and augmentation of the BMD. It also should be noted that, unlike post-menopausal women, the young adult rats used in this work were still growing, which is a distinct situation in the clinical administration of TPTD; however, the vertebral bodies analyzed in this work did not show obvious outgrowth, as evidenced by the observations in the sham/vehicle group. Therefore, further studies in animals with bone remodeling, such as rabbits, should be informative.

In the present study, however, we observed that an increased administration frequency as well as dosage of TPTD-induced cortical porosity was associated with an increase in the levels of systemic bone resorption markers. This finding was likely due to the fact that a prolonged or persistent PTH level at a given threshold in blood stream constitutively stimulated the long CAMP signaling, thereby causing cortical porosity that involved site-specific intracortical bone resorption, an increased number of osteocytes, and possible osteocytic osteolysis.

In conclusion, our regimens of TPTD in a standard OVX rat model of post-menopausal osteoporosis showed that the development of cortical porosity was associated with high administration frequency as well as the dosage, which is plausibly explained by constitutively activated endosome-mediated intracellular signaling by TPTD in osteoblast-lineage cells. Furthermore, the combined analysis of three-dimensional micro-CT and successive deconvolution fluorescence tiling imaging in this study enabled us to manifest the spatial development of cortical porosity in a consistent manner from the whole-tissue to cellular level. Our observations suggested that the distinction of the mechanical properties at local bone sites was a critical factor for developing cortical porosity. The association between the duration of CAMP signaling and the 
downstream responses that promote bone anabolism and catabolism, which could also unravel the PTH paradox of intermittent versus continuous administration, should be investigated in future studies. These molecular pathways may be affected by mechanical loading and unloading to bone cells. Further investigation and consideration are still needed to extrapolate clinical situation from data obtained from animal models in terms of the development of cortical porosity.

\section{Acknowledgements}

This study was funded by the Asahi Kasei Pharma Corporation. We thank Hiroshi Kuriyama, VMD., for providing scientific advice, and Ms. Masako Onotogi from Ina Research for assisting with the animal management. This work was also partly supported by JSPS KAKENHI grant numbers 16K20412 and 26293392 to J-W. Lee and T. Iimura, respectively. This work was also partly supported by The Institute of Medical Science, The University of Tokyo: IMSUT Joint Research Project (2014-241). We express sincere thanks to Kazuaki Tokunaga, Satoshi Takiguchi, Minoru Harada and Norio Ohba at Nikon Instech, Ltd. for their valuable expertise on microscopy and image processing, and to Noriko Tokai and Miwako Iwai at IMUST for their expertise on microscopy.

\section{Competing interests}

Aya Shimomura, Ryoko Takao-Kawabata, Kyoko Hirano, Yukihiro Isogai, and Toshinori Ishizuya are employees of Asahi Kasei Pharma Corporation. The remaining authors declare no conflict of interest.

\section{References}

1 Albright F, Bauer W, Ropes M et al. Studies of calcium and phosphorus metabolism: IV. The effect of the parathyroid hormone. J Clin Invest 1929; 7: 139-181.

2 Burrows RB. Variations produced in bones of growing rats by parathyroid extracts. Am J Anat 1938; 62: 237-290.

3 Pugsley LI, Selye H. The histological changes in the bone responsible for the action of parathyroid hormone on the calcium metabolism of the rat. J Physiol 1933; 79: 113-117.

4 Parsons JA, Rafferty B, Stevenson RW et al. Evidence that protease inhibitors reduce the degradation of parathyroid hormone and calcitonin injected subcutaneously. Br J Pharmacol 1979; 66: 25-32.

5 Reeve J, Meunier PJ, Parsons JA et al. Anabolic effect of human parathyroid hormone fragment on trabecular bone in involutional osteoporosis: a multicentre trial. Br Med J 1980; 280: 1340-1344.

6 Etoh M, Yamaguchi A. Repetition of continuous PTH treatments followed by periodic withdrawals exerts anabolic effects on rat bone. J Bone Miner Metab 2010; 28: 641-649.

7 Jilka RL. Molecular and cellular mechanisms of the anabolic effect of intermittent PTH. Bone 2007; 40: 1434-1446.

8 Baron R, Hesse E. Update on bone anabolics in osteoporosis treatment: rationale, current status, and perspectives. J Clin Endocrinol Metab 2012; 97: 311-325.

9 Cheloha RW, Gellman SH, Vilardaga JP et al. PTH receptor-1 signallingmechanistic insights and therapeutic prospects. Nat Rev Endocrinol 2015; 11: 712-724.

10 Cheloha RW, Maeda A, Dean T et al. Backbone modification of a polypeptide drug alters duration of action in vivo. Nat Biotechnol 2014; 32: 653-655.
11 Okazaki M, Ferrandon S, Vilardaga JP et al. Prolonged signaling at the parathyroid hormone receptor by peptide ligands targeted to a specific receptor conformation. Proc Natl Acad Sci USA 2008; 105: 16525-16530.

12 Diab T, Condon KW, Burr DB et al. Age-related change in the damage morphology of human cortical bone and its role in bone fragility. Bone 2006; 38: 427-431.

13 Bala Y, Zebaze R, Seeman E. Role of cortical bone in bone fragility. Curr Opin Rheumatol 2015; 27: 406-413.

14 Bandeira L, Bilezikian J. Primary hyperparathyroidism. F1000Res 2016; 5: F1000 Faculty Rev-1.

15 Zebaze RM, Ghasem-Zadeh A, Bohte A et al. Intracortical remodelling and porosity in the distal radius and post-mortem femurs of women: a cross-sectional study. Lancet 2010; 375: 1729-1736.

16 Stein EM, Silva BC, Boutroy S et al. Primary hyperparathyroidism is associated with abnormal cortical and trabecular microstructure and reduced bone stiffness in postmenopausal women. J Bone Miner Res 2013; 28: 1029-1040.

17 Shigdel R, Osima M, Ahmed LA et al. Bone turnover markers are associated with higher cortical porosity, thinner cortices, and larger size of the proximal femur and non-vertebral fractures. Bone 2015; 81: 1-6.

18 Yeni YN, Brown CU, Wang Z et al. The influence of bone morphology on fracture toughness of the human femur and tibia. Bone 1997; 21: 453-459.

19 Uzawa T, Hori M, Ejiri S et al. Comparison of the effects of intermittent and continuous administration of human parathyroid hormone(1-34) on rat bone. Bone 1995; 16: 477-484.

20 Hansen S, Hauge EM, Beck Jensen JE et al. Differing effects of PTH 1-34, PTH 1-84, and zoledronic acid on bone microarchitecture and estimated strength in postmenopausal women with osteoporosis: an 18-month open-labeled observational study using HR-pQCT. J Bone Miner Res 2013; 28: 736-745.

21 Hirano T, Burr DB, Cain RL et al. Changes in geometry and cortical porosity in adult, ovary-intact rabbits after 5 months treatment with LY333334 (hPTH 1-34). Calcif Tissue Int 2000; 66: 456-460.

22 Inoue J. Bone changes with long term administration of low dose 1-34 human PTH on adult beagles. Nihon Seikeigeka Gakkai Zasshi 1985; 59: 409-427.

23 Takao-Kawabata R, Isogai Y, Takakura A et al. Three-times-weekly administration of teriparatide improves vertebral and peripheral bone density, microarchitecture, and mechanical properties Without accelerating bone resorption in ovariectomized rats. Calcif Tissue Int 2015; 97 : 156-168.

24 Sugie-Oya A, Takakura A, Takao-Kawabata R et al. Comparison of treatment effects of teriparatide and the bisphosphonate risedronate in an aged, osteopenic, ovariectomized rat model under various clinical conditions. J Bone Miner Metab 2015; 34: 303-314.

25 Isogai Y, Takao-Kawabata R, Takakura A et al. Early effects of single and low-frequency repeated administration of teriparatide, $\mathrm{hPTH}(1-34)$, on bone formation and resorption in ovariectomized rats. Calcif Tissue Int 2015; 97: 412-420.

26 Tanizawa T, Yamaguchi A, Uchiyama $Y$ et al. Reduction in bone formation and elevated bone resorption in ovariectomized rats with special reference to acute inflammation. Bone 2000; 26: 43-53.

27 Takakura A, Takao-Kawabata R, Isogai Y et al. Differences in vertebral, tibial, and iliac cancellous bone metabolism in ovariectomized rats. J Bone Miner Metab 2015; 34: 291-302.

28 Mosekilde L, Danielsen CC, Knudsen UB. The effect of aging and ovariectomy on the vertebral bone mass and biomechanical properties of mature rats. Bone 1993; 14: 1-6. 
29 Parfitt AM, Drezner MK, Glorieux FH et al. Bone histomorphometry: standardization of nomenclature, symbols, and units. Report of the ASBMR Histomorphometry Nomenclature Committee. J Bone Miner Res 1987; 2: 595-610.

30 Dempster DW, Compston JE, Drezner MK et al. Standardized nomenclature, symbols, and units for bone histomorphometry: a 2012 update of the report of the ASBMR Histomorphometry Nomenclature Committee. J Bone Miner Res 2013; 28: 2-17.

31 Watanabe T, Tamamura Y, Hoshino A et al. Increasing participation of sclerostin in postnatal bone development, revealed by three-dimensional immunofluorescence morphometry. Bone 2012; 51: 447-458.

32 Tsurukami H, Nakamura T, Suzuki K et al. A novel synthetic vitamin D analogue, 2 beta-(3-hydroxypropoxy)1 alpha, 25-dihydroxyvitamin D3 (ED-71), increases bone mass by stimulating the bone formation in normal and ovariectomized rats. Calcif Tissue Int 1994; 54: 142-149.

33 Miki T, Nakatsuka K, Naka H et al. Effect and safety of intermittent weekly administration of human parathyroid hormone 1-34 in patients with primary osteoporosis evaluated by histomorphometry and microstructural analysis of iliac trabecular bone before and after 1 year of treatment. J Bone Miner Metab 2004; 22: 569-576.

34 Chavassieux PM, Arlot ME, Reda C et al. Histomorphometric assessment of the long-term effects of alendronate on bone quality and remodeling in patients with osteoporosis. J Clin Invest 1997; 100: 1475-1480.

35 Ste-Marie LG, Sod E, Johnson T et al. Five years of treatment with risedronate and its effects on bone safety in women with postmenopausal osteoporosis. Calcif Tissue Int 2004; 75: 469-476.

36 Ibbotson KJ, Orcutt CM, D'Souza SM et al. Contrasting effects of parathyroid hormone and insulin-like growth factor I in an aged ovariectomized rat model of postmenopausal osteoporosis. J Bone Miner Res 1992; 7: 425-432.

37 Lauritzen DB, Balena R, Shea $\mathrm{M}$ et al. Effects of combined prostaglandin and alendronate treatment on the histomorphometry and biomechanical properties of bone in ovariectomized rats. J Bone Miner Res 1993; 8: 871-879.

38 Zhou H, Shen V, Dempster DW et al. Continuous parathyroid hormone and estrogen administration increases vertebral cancellous bone volume and cortical width in the estrogen-deficient rat. J Bone Miner Res 2001; 16: 1300-1307.

39 Sietsema WK. Animal models of cortical porosity. Bone 1995; 17: 297S-305S.

40 O'Brien CA, Plotkin LI, Galli C et al. Control of bone mass and remodeling by PTH receptor signaling in osteocytes. PLoS ONE 2008; 3 : e2942.

41 Rhee Y, Allen MR, Condon K et al. PTH receptor signaling in osteocytes governs periosteal bone formation and intracortical remodeling. J Bone Miner Res 2011; 26: 1035-1046.

42 Tanaka S, Sakai A, Tanaka M et al. Skeletal unloading alleviates the anabolic action of intermittent PTH(1-34) in mouse tibia in association with inhibition of PTH-induced increase in c-fos mRNA in bone marrow cells. J Bone Miner Res 2004; 19: 1813-1820.
43 Grosso MJ, Courtland HW, Yang X et al. Intermittent PTH administration and mechanical loading are anabolic for periprosthetic cancellous bone. J Orthop Res 2015; 33: 163-173.

44 Himeno-Ando A, Izumi Y, Yamaguchi A et al. Structural differences in the osteocyte network between the calvaria and long bone revealed by three-dimensional fluorescence morphometry, possibly reflecting distinct mechano-adaptations and sensitivities. Biochem Biophys Res Commun 2012; 417: 765-770.

45 Sugawara Y, Kamioka H, Ishihara Y et al. The early mouse 3D osteocyte network in the presence and absence of mechanical loading. Bone 2013; 52: 189-196.

46 Dallas SL, Bonewald LF. Dynamics of the transition from osteoblast to osteocyte. Ann NY Acad Sci 2010; 1192: 437-443.

47 Palumbo C, Palazzini S, Marotti G. Morphological study of intercellular junctions during osteocyte differentiation. Bone 1990; 11: 401-406.

48 Palumbo C, Palazzini S, Zaffe D et al. Osteocyte differentiation in the tibia of newborn rabbit: an ultrastructural study of the formation of cytoplasmic processes. Acta Anat 1990; 137: 350-358.

49 Piemontese M, Onal M, Xiong J et al. Low bone mass and changes in the osteocyte network in mice lacking autophagy in the osteoblast lineage. Sci Rep 2016; 6: 24262.

50 Tazawa K, Hoshi K, Kawamoto S et al. Osteocytic osteolysis observed in rats to which parathyroid hormone was continuously administered. J Bone Miner Metab 2004; 22: 524-529.

51 Nango N, Kubota S, Hasegawa T et al. Osteocyte-directed bone demineralization along canaliculi. Bone 2016; 84: 279-288.

52 Qing H, Ardeshirpour L, Pajevic PD et al. Demonstration of osteocytic perilacunar/canalicular remodeling in mice during lactation. J Bone Miner Res 2012; 27: 1018-1029.

53 Shiraki M, Sugimoto T, Nakamura T. Effects of a single injection of teriparatide on bone turnover markers in postmenopausal women. Osteoporos Int 2013; 24: 219-226.

54 Sugimoto T, Nakamura T, Nakamura Y et al. Profile of changes in bone turnover markers during once-weekly teriparatide administration for 24 weeks in postmenopausal women with osteoporosis. Osteoporos Int 2014; 25: 1173-1180.

55 Serada M, Sakurai-Tanikawa A, Igarashi M et al. The role of the liver and kidneys in the pharmacokinetics of subcutaneously administered teriparatide acetate in rats. Xenobiotica 2012; 42: 398-407.

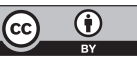

This work is licensed under a Creative Commons Attribution 4.0 International License. The images or other third party material in this article are included in the article's Creative Commons license, unless indicated otherwise in the credit line; if the material is not included under the Creative Commons license, users will need to obtain permission from the license holder to reproduce the material. To view a copy of this license, visit http:/ / creativecommons.org/licenses/by/4.0/

(C) The Author(s) 2017

Supplementary Information for this article can be found on the Bone Research website (http://www.nature.com/boneres) 